\title{
Intense velocity-shears, magnetic fields and filaments in diffuse gas
}

\author{
Edith Falgarone ${ }^{1}$, Pierre Hily-Blant ${ }^{2}$, François Levrier ${ }^{1}$, \\ Manuel Berthet ${ }^{1}$, Pierre Bastien ${ }^{3}$ and Dan Clemens ${ }^{4}$ \\ ${ }^{1}$ LERMA/LRA, Ecole Normale Supérieure \& Observatoire de Paris, \\ 24 rue Lhomond, 75005 Paris, France \\ email: edith.falgarone@ens.fr \\ ${ }^{2}$ IPAG, Observatoire de Grenoble, St Martin d'Hères, France \\ ${ }^{3}$ University of Montréal, Montréal, Canada \\ ${ }^{4}$ Institute for Astrophysical Research, Boston University, Boston, USA
}

\begin{abstract}
The dissipation of turbulence is a key process in the evolution of diffuse gas towards denser structures. The vast range of coupled scales and the variety of dissipative processes in interstellar turbulence make it a complex system to analyze. Observations now provide powerful statistics of the gas velocity field, density and magnetic field orientations, opening a rich field of investigation. On-going comparisons of the orientation of intense velocity-shears, magnetic field and tenuous filaments of matter in a turbulent high-latitude cloud are promising.
\end{abstract}

Keywords. Turbulence, Magnetic Fields, ISM: molecules, ISM: kinematics and dynamics, ISM: general, ISM: evolution

Turbulent dissipation is intermittent in space and time and it is now identified in the ISM via the non-Gaussian statistics of the velocity field. Parsec-scale coherent structures of intense velocity-shears have been found in the CO line emission of a diffuse molecular cloud in the Polaris Flare (Hily-Blant et al. 2008). One of them is structured into narrow shear-layers down to the milliparsec-scale with straight projections on the plane of the sky (POS) and widely different orientations (Falgarone et al. 2009). Each of the main shear orientations in the mpc-field can be found in the pc-scale structures.

The POS projections of the magnetic field have been studied via the polarization of starlight: (i) at the $30 \mathrm{pc}$-scale, in the visible, with the Beauty and the Beast polarimeter. The distribution of the 50 position-angles (PA) in the $30 \mathrm{pc}$ field is also broad, (ii) at the $0.1 \mathrm{pc}-$-scale with the Mimir polarimeter (Clemens et al., 2012) in the near IR. Interestingly the 7 measured polarization PAs cover the same broad range as those of the $30 \mathrm{pc}$ field, and a few of them are parallel to the local structure of intense velocity-shear. Last, the orientations of the striations in dust emission maps of the large scale field have also been studied. The remarkable similarity of the three PA distributions (magnetic field, velocity-shears and dust filaments) supports a close connection not only between large and small scales but also between the topology of $\mathbf{B}$ with that of the most dissipative structures and tenuous filaments of matter. Recent Herschel/SPIRE observations further support this view.

\section{References}

Clemens, D. P., Pinnick, A. F. \& Pavel, M. D., 2012 ApJS, 200, 20

Falgarone, E., Pety, J., \& Hily-Blant, P., 2009, A\&AA, 507, 355

Hily-Blant, P., Falgarone, E. \& Pety, J., 2008 A $\& A$ 481, 367 